\title{
ALMOST POWER SUM SYSTEMS
}

\author{
V. I. KOROBOV AND A. N. BUGAEVSKAYA
}

\begin{abstract}
We consider the system of nonlinear almost polynomial equations without power gaps or with even power gaps. The method is to reduce the given system to finding the roots of univariate functions, some of which are polynomials. For the system with even power gaps the obtained equalities are the analogs of Newton's identities. These equalities express the connection between elementary symmetric functions and odd power sums.
\end{abstract}

\section{INTRODUCTION}

The main objective of this paper is construction of methods for solving the systems of nonlinear equations of the forms

$$
\begin{aligned}
& -\sum_{i=1}^{m} T_{i}^{k}+\sum_{i=m+1}^{n-1} T_{i}^{k}+f_{k}\left(\varphi\left(T_{1}, \ldots, T_{n}\right)\right)=0, \quad k=1, \ldots, n, \\
& -\sum_{i=1}^{m} T_{i}^{2 k-1}+\sum_{i=m+1}^{n-1} T_{i}^{2 k-1}+f_{2 k-1}\left(\varphi\left(T_{1}, \ldots, T_{n}\right)\right)=0, k=1, \ldots, n,
\end{aligned}
$$

where $\varphi\left(T_{1}, \ldots, T_{n}\right), f_{1}(\varphi), \ldots, f_{n}(\varphi)$ and $f_{1}(\varphi), f_{3}(\varphi), \ldots, f_{2 n-1}(\varphi)$ are given continuous functions of their arguments. We call the systems of the form (1.1) the systems of almost power-sum equations and (1.2) the systems of almost power-sum equations with even power gaps. As an example one might consider the system of equations

$$
\left\{\begin{array}{l}
-T_{1}+T_{1}^{2}-T_{2}+T_{3}+2 T_{1} T_{4}+T_{4}^{2}-4=0 \\
-T_{1}^{3}-T_{2}^{3}+T_{3}^{3}+3 \sin \frac{\pi\left(T_{1}+T_{4}\right)}{2}+6=0 \\
-T_{1}^{5}-T_{2}^{5}+T_{3}^{5}-2 \cos \pi\left(T_{1}+T_{4}\right)+32=0 \\
-T_{1}^{7}-T_{2}^{7}+T_{3}^{7}+126=0
\end{array}\right.
$$

we give detailed analysis of this system in Section 4 .

First we give expressions for odd power sums $\sum_{i=1}^{n} T_{i}^{2 k-1}, k=1,2, \ldots$, in terms of elementary symmetric functions. These formulas are analogs of well-known Newton identities, expressing relations between power sums $\sum_{i=1}^{n} T_{i}^{k}, k=1,2, \ldots$, and of elementary symmetric polynomials. Let $S_{k}:=\sum_{i=1}^{n} T_{i}^{k}$; we will use $S_{k}$ for this

Received by the editor July 10, 2013 and, in revised form, February 4, 2014, July 11, 2014, and August 27, 2014.

2010 Mathematics Subject Classification. Primary 65H10, 65D32; Secondary 30E05, 49N05.

Key words and phrases. Systems of almost polynomial equations, moment problem, elementary symmetric functions, power sums, quadrature formulas. 
expression, and if numbers are substituted for $T_{1}, \ldots, T_{n}$, then we express the resulting number by $s_{k}$.

For the systems of nonlinear equations of the form $F_{i}\left(T_{1}, \ldots, T_{n}\right)=0, i=$ $1, \ldots, n$, there are many different numerical methods available for solving them, among which the iterative methods are the most common. On the other hand, solving this system can be reduced to solving for the minimum of an appropriate function; of course, these two problems are of the same numerical complexity.

For the case when functions $F_{i}\left(T_{1}, \ldots, T_{n}\right), i=1, \ldots, n$, are polynomials, many methods of solving the connected systems of equations have been developed over the last three decades. One of the basic methods is an approach based on reducing the original problem to the problem of finding the roots of some univariate polynomials. Such polynomials may be obtained, for example, by using resultants or Gröbner bases, but the resulted polynomials are often of pretty high degrees. However, for polynomials of special type, it may be possible to obtain the equivalent univariate polynomial equations of considerably low degrees, $n$ in many cases. Moreover, there arise the questions of existence of real and positive roots or the roots in a given interval.

A classical example is the system of the form

$$
\sum_{i=1}^{n} T_{i}^{k}=s_{k}, \quad k=1, \ldots, n,
$$

where the numbers $s_{1}, \ldots, s_{n}$ are given and $T_{1}, \ldots, T_{n}$ are unknowns. This system can be solved easily by utilizing the so-called "Newton's identities". The system of equations (1.3) arises, for instance, in searching for the coefficients of the characteristic polynomial of an unknown matrix $A$ under the assumption that the traces $s_{k}$ of matrices $A^{k}(k=1, \ldots, n)$ are given (see, e.g. Le Verrier [1]).

The system of the form (1.3) appears also when deriving the quadrature formulas of Chebyshev type [2],

$$
\int_{a}^{b} p(t) f(t) d t=A \sum_{i=1}^{n} f\left(T_{i}\right) .
$$

Here $p(t)>0$ is the given weight function and the unknown numbers $T_{k} \in[a, b], k=$ $1, \ldots, n$ are called the nodes of the quadrature formula. The number $A$ and the nodes $T_{1}, \ldots, T_{n}$ are to be selected so that the quadrature formula (1.4) holds exactly for the functions $f(t)=1, t, t^{2}, \ldots, t^{n}$. In the case of $a=-1, b=1$, $p(t) \equiv 1$ we obtain the Chebyshev quadrature formula where the nodes can be found as the roots of the following polynomial system of equations:

$$
\sum_{i=1}^{n} T_{i}^{k}=\frac{n\left(1+\left(-1^{k}\right)\right)}{2(k+1)}, \quad k=1, \ldots, n .
$$

Moreover, S. N. Bernstein had established in [3] that for $n=8$ and $n \geq 10$ the above polynomial system has complex roots. If $a=-1, b=1, p(t)=\frac{1}{\sqrt{1-t^{2}}}$, then (1.4) was first obtained by Mehler [4],

$$
\int_{-1}^{1} \frac{f(t)}{\sqrt{1-t^{2}}} d t \cong \frac{\pi}{n} \sum_{i=1}^{n} f\left(T_{i}\right)
$$


where the nodes satisfy the polynomial system

$$
\sum_{i=1}^{n} T_{i}^{k}=\frac{1+(-1)^{k}}{2} \frac{(k-1) ! !}{k ! !} n, \quad k=1, \ldots, n,
$$

which yields $T_{i}=\cos \frac{(2 i-1) \pi}{2 n}, i=1, \ldots, n$, the roots of the Chebyshev polynomials of the first kind $P_{n}(x)=\cos (n \arccos x), x \in[-1,1]$. This formula is also called the Chebyshev-Gauss quadrature. In [5] J. L. Ullman established that if

$$
p(t)=\frac{1}{\sqrt{1-t^{2}}} \frac{1+2 a t}{1+4 a^{2}+4 a t}, \quad|a| \leq \frac{1}{4},
$$

then the polynomial system for finding the nodes of the Chebyshev quadrature formula has real roots.

Another example is the polynomial system arising in the power moment problem. To see this, let us consider the so-called Markov power moment min-problem, which can be stated as follows. For a given sequence of functions $\left\{t^{k-1}\right\}_{k=1}^{n}, t \in[0, T]$ and given vector-row $s=\left(s_{1}, \ldots, s_{n}\right) \in \mathbb{R}^{n}$ find the smallest possible closed interval $[0, \theta] \subset[0, T]$ and a function $u(t)$ such that the following expressions hold:

$$
s_{k}=\int_{0}^{\theta} t^{k-1} u(t) d t, \quad k=1, \ldots, n ; \quad|u(t)| \leq 1 \text { for } t \in[0, \theta] .
$$

The Markov power moment min-problem was formulated and solved by V. I. Korobov and G. M. Sklyar first in 1987-1989 in [6,7] and has been studied further in their many subsequent works (see, e.g. [8,9]). In particular, it was shown that the above power moment min-problem is equivalent to the time-optimal control problem for the "canonical" linear system and the analytical solving of such a problem can be reduced to finding the roots of a power polynomial system. In fact, let $(\theta, u(t))$ be a solution of the problem (1.6) with the minimum $\theta>0$. Then it is known that $u(t)$ is a piecewise-constant function which takes values \pm 1 and has no more than $n-1$ points of discontinuity on the interval $(0, \theta)$. If we denote the points of discontinuity by $T_{1}, \ldots, T_{n-1}, 0<T_{1}<\cdots<T_{n-1}<\theta=T_{n}$, then from (1.6) it follows easily that $T_{1}, \ldots, T_{n}$ satisfy the system of polynomial equations of the form

$$
\sum_{i=1}^{n-1}(-1)^{i+1} T_{i}^{k}+\frac{(-1)^{n+1}}{2} T_{n}^{k}= \pm \frac{k}{2} s_{k}, k=1, \ldots, n,
$$

where the signs \pm of $s_{k}$ on the right-hand side of the above equations correspond to the cases when function $u(t)$ takes, respectively, values +1 or -1 on the interval $\left(0, T_{1}\right)$. Developing this method, in [10] in particular, a min-moment problem with even gaps was considered, and it was solved using a polynomial system of equations similar to (1.7) but with even gaps.

We note that methods of solving polynomial systems of the form

$$
\sum_{i=1}^{n} c_{i} x_{i}^{j}=s_{j}, \quad j=1,2, \ldots, n, \quad c_{i} \in\{0, \pm 1\}
$$

have been developed in [6]-[13] in connection with the investigation of moment problems. For polynomial systems of the form

$$
\sum_{i=1}^{n-1} x_{i}^{j}-\alpha x_{n}^{j}=s_{j}, \quad j=1,2, \ldots, n,
$$


where $\alpha$ is an arbitrary number, some solving methods which use Newton's identities have been presented in 14 .

Beside independent interest to solve (1.2), many problems can be reduced to systems of such a form, as shown in the following examples. First, these systems obviously appear in the modified Le Verrier problem of searching for the eigenvalues of the matrix $A$ when the traces of odd powers of the matrix $A$, i.e., the traces of matrices $A, A^{3}, \ldots, A^{2 p-1}$ are given. Further, let us consider the construction of the quadrature formulas of Chebyshev type with Ullman weight (1.5) for the functions of the form $f(t)=B+\varphi(t)$, where $B$ is a constant, $\varphi(t)$ is an odd function and $t \in[a, b]$. We require that quadrature formula hold exactly for the functions $f(t)=1, t, t^{3}, \ldots, t^{2 n-1}$, i.e.,

$$
\int_{a}^{b} p(t) d t=n A, \quad \int_{a}^{b} p(t) t^{2 k-1} d t=A \sum_{i=1}^{n} T_{i}^{2 k-1}, \quad k=1, \ldots, n .
$$

If $p(t) \equiv 1$, then we will call this quadrature formula quadrature formula of Chebyshev type with even gaps, and if $p(t)=1 / \sqrt{1-t^{2}}$ (for $t \in[0,1]$ ) then we will call it quadrature formula of Mehler type with even gaps.

In this case the problem of finding nodes of the quadrature formula, as in the case of the Le Verrier problem, will be reduced to solving the following polynomial system with the even gaps but without the alternation of signs:

$$
\sum_{i=1}^{n} T_{i}^{2 k-1}=s_{2 k-1}, k=1, \ldots, n .
$$

We notice additionally that $s_{2 k-1}=n /(2 k)$ for the Chebyshev quadrature formula, and $s_{2 k-1}=2 n(2 k-2) ! ! /(\pi(2 k-1) ! !)$ for the Mehler quadrature formula.

We note that the solution of the system (1.3) is unique (up to permutation of variables). On the other hand, the system (1.2) and, in particular, the system (1.8), may have a unique solution (up to permutation of the variable), as well as an infinite number of solutions, and a solution may not exist at all.

Finally, the polynomial system with the even gaps and with the alternation of signs of $T_{1}, \ldots, T_{n}$ arises in solving the power moment min-problem: find the minimal time $\theta=T>0$ and the function $u(t)(0 \leq t \leq T)$ satisfying

$$
\int_{0}^{T} t^{2 k-2} u(t) d t=s_{2 k-1}, \quad|u(t)| \leq 1, k=1, \ldots, n .
$$

Similarly to the case of problem (1.6), the min-problem (1.9) is equivalent to the time-optimal control problem for the system

$$
\dot{x}=A x+b u, \quad|u| \leq 1,
$$

where the constant matrix $A$ has the spectrum $\sigma(A)=\{\lambda, 3 \lambda, \ldots,(2 n-1) \lambda\}$. The optimal solution $u(t)$ of (1.9) is a piecewise constant function with values \pm 1 and with the points of discontinuity $T_{1}, \ldots, T_{n-1}, T_{n}=T$ satisfying the following polynomial system (with even gaps):

$$
\sum_{i=1}^{n-1}(-1)^{n+i+1} T_{i}^{2 k-1}-\frac{T^{2 k-1}}{2}=s_{2 k-1}, \quad k=1, \ldots, n .
$$

In this paper we will first consider the polynomial system with even gaps (1.8) and develop two methods for solving this system. The first method consists in 
establishing relations between the elementary symmetric polynomials

$$
\begin{aligned}
\sigma_{1} & =T_{1}+T_{2}+\cdots+T_{n}, \\
\sigma_{2} & =T_{1} T_{2}+T_{1} T_{3}+\cdots+T_{n-1} T_{n}, \\
\sigma_{3} & =T_{1} T_{2} T_{3}+T_{1} T_{2} T_{4}+\cdots+T_{n-2} T_{n-1} T_{n}, \\
& \vdots \\
\sigma_{n} & =T_{1} T_{2} \ldots T_{n},
\end{aligned}
$$

and the odd powers sums $\sum_{i=1}^{n} T_{i}^{2 k-1}, k=1,2, \ldots, \infty$ in terms of equalities which are analogs of Newton's identities. By using these analogs of Newton's identities it is possible to reduce the problem of solving system (1.8) to finding the roots of a univariate polynomial. This method will be presented in Sections 2 and 3, where some examples will be given to illustrate its use. The second method is more general and used to solve systems of nonlinear equations of the form (1.1)(1.2). This method is used to reduce finding unknowns $T_{1}, \ldots, T_{n}$ to the successive determination of the roots of univariate functions. Moreover, as will be shown in Section 4, in the case of "almost polynomial" systems with even gaps, the number of such functions does not exceed four, two of which are polynomials. Finally, the problem of solving "almost polynomial" systems without gaps is considered in Section 5 and is shown to be equivalent to finding the roots of four univariate functions.

To simplify the notation later on we will denote a Hankel matrix by

$$
\Gamma_{i, k}^{j}=\left(\begin{array}{cccc}
\gamma_{i} & \gamma_{i+j} & \ldots & \gamma_{i+(k-1) j} \\
\gamma_{i+j} & \gamma_{i+2 j} & \ldots & \gamma_{i+k j} \\
\vdots & \vdots & \ddots & \vdots \\
\gamma_{i+(k-1) j} & \gamma_{i+k j} & \ldots & \gamma_{i+2(k-1) j}
\end{array}\right),
$$

and Hankel determinants by

$$
\Delta_{i, k}=\operatorname{det}\left(\Gamma_{i, k}^{1}\right), \tilde{\Delta}_{i, k}=\operatorname{det}\left(\Gamma_{i, k}^{2}\right) .
$$

\section{SURVEY OF SYSTEMS OF POWER-SUMS}

Although Newton's identities are well known, we show an expression which gives us Newton's identities, because similar expressions will be used later:

$$
\ln \left(1+\sum_{k=1}^{n} \frac{(-1)^{k} \sigma_{k}}{z^{k}}\right)=-\sum_{k=1}^{\infty} \frac{S_{k}}{k z^{k}}
$$

This equality can be obtained as follows. We have

$$
\ln \left(1-\frac{T_{1}}{z}\right)+\cdots+\ln \left(1-\frac{T_{n}}{z}\right)=\ln \left(1-\frac{T_{1}}{z}\right) \cdots\left(1-\frac{T_{n}}{z}\right)=\ln \left(1+\sum_{k=1}^{n} \frac{(-1)^{k} \sigma_{k}}{z^{k}}\right) .
$$

Expanding the left-hand side of this expression into Taylor series in $1 / z$ and collecting terms of the same powers, we obtain the right-hand side expression of (2.1). 
On the other hand, differentiating the equality (2.1) with respect to $1 / z$, we obtain the following relation:

$$
\frac{\sum_{k=1}^{n} \frac{(-1)^{k} k \sigma_{k}}{z^{k-1}}}{1+\sum_{k=1}^{n} \frac{(-1)^{k} \sigma_{k}}{z^{k}}}=-\sum_{k=1}^{\infty} \frac{S_{k}}{z^{k-1}} .
$$

Comparing coefficients with the same powers of $z$ in the equality (2.2) we obtain Newton's identities

$$
\begin{aligned}
& S_{k}-\sigma_{1} S_{k-1}+\sigma_{2} S_{k-2}-\cdots+(-1)^{k} k \sigma_{k}=0 \text { for } 1 \leq k \leq n, \\
& S_{k}-\sigma_{1} S_{k-1}+\sigma_{2} S_{k-2}-\cdots+(-1)^{n} \sigma_{n} S_{k-n}=0 \text { for } k>n, \\
& S_{0}=1, \quad S_{k}=0 \text { for } k<0,
\end{aligned}
$$

that establish the connection between the power sums and the elementary symmetric functions.

Explicit expressions for elementary symmetric functions $\sigma_{1}, \ldots, \sigma_{n}$ in terms of sums $S_{1}, \ldots, S_{n}$ and, vice versa, are given by well-known Waring formulas, where the summations below are over all sets of nonnegative integers $\lambda_{1}, \ldots, \lambda_{k}$ for which $\lambda_{1}+2 \lambda_{2}+\cdots+k \lambda_{k}=k$ :

$$
(-1)^{k} \sigma_{k}=\sum \frac{(-1)^{\lambda_{1}+\cdots+\lambda_{k}} S_{1}^{\lambda_{1}} \ldots S_{k}^{\lambda_{k}}}{1^{\lambda_{1}} 2^{\lambda_{2}} \ldots k^{\lambda_{k}} \lambda_{1} ! \ldots \lambda_{k} !}, \quad k=1, \ldots, n
$$

and

$$
S_{k}=k \sum(-1)^{k+\lambda_{1}+\ldots+\lambda_{k}} \frac{\left(\lambda_{1}+\cdots+\lambda_{k}-1\right) !}{\lambda_{1} ! \ldots \lambda_{k} !} \sigma_{1}^{\lambda_{1}} \ldots \sigma_{k}^{\lambda_{k}}, k=1,2, \ldots, \infty .
$$

Solving $n$ equations in (2.3) w.r.t. $\sigma_{1}, \ldots, \sigma_{n}$ (resp. $S_{1}, \ldots, S_{n}$ ), we obtain the following determinants expressions for $\sigma_{k}$ (resp. $\left.S_{k}\right)$ :

$$
\begin{gathered}
\sigma_{k}=\sigma_{k}\left(S_{1}, \ldots, S_{k}\right)=\frac{1}{k !}\left|\begin{array}{ccccc}
S_{1} & 1 & 0 & \ldots & 0 \\
S_{2} & S_{1} & 2 & \ldots & 0 \\
\vdots & \vdots & \vdots & \ddots & \vdots \\
S_{k-1} & S_{k-2} & S_{k-3} & \ldots & k-1 \\
S_{k} & S_{k-1} & S_{k-2} & \ldots & S_{1}
\end{array}\right|, k=1, \ldots, n, \\
\quad S_{k}=\left|\begin{array}{ccccc}
\sigma_{1} & 2 \sigma_{2} & \ldots & (k-1) \sigma_{k-1} & k \sigma_{k} \\
1 & \sigma_{1} & \ldots & \sigma_{k-2} & \sigma_{k-1} \\
\vdots & \vdots & \ddots & \vdots & \vdots \\
0 & 0 & \ldots & 1 & \sigma_{1}
\end{array}\right|, k=1,2, \ldots, n .
\end{gathered}
$$

Suppose now that power sums $\sum_{i=1}^{n} T_{i}^{k}, k=1, \ldots, n$, are given numbers $s_{1}, \ldots, s_{n}$, we want to find the values of $T_{1}, \ldots, T_{n}$ which satisfy the equations

$$
\sum_{i=1}^{n} T_{i}^{k}=s_{k}, \quad k=1, \ldots, n .
$$


Using the equalities (2.4), the system of polynomial equations (1.3) with respect to $T_{1}, \ldots, T_{n}$ is reduced to the equation

$$
t^{n}-\sigma_{1} t^{n-1}+\cdots+(-1)^{n} \sigma_{n}=0
$$

that has roots $T_{1}, \ldots, T_{n}$.

We present another way of finding a polynomial equation (2.6), which we use later on. Consider the system

$$
\sum_{i=1}^{n} T_{i}^{k}=s_{k}, \quad n=1,2, \ldots, \infty
$$

where $s_{1}, \ldots, s_{n}$ are given numbers. The system of equations (2.7) is symmetric with respect to the variables $T_{1}, \ldots, T_{n}$. We denote by $T$ any of those variables; for simplicity of our notation let $T=T_{n}$. We write the system (2.7) in the form

$$
\sum_{i=1}^{n-1} T_{i}^{k}=s_{k}-T^{k}, \quad k=1,2, \ldots, \infty .
$$

This system is obtained from the equality

$$
\ln \left(1-\frac{T_{1}}{z}\right) \ldots\left(1-\frac{T_{n-1}}{z}\right)=\ln \left(1+\sum_{k=1}^{n-1} \frac{(-1)^{k} \bar{\sigma}_{k}}{z^{k}}\right)=-\sum_{k=1}^{\infty} \frac{s_{k}-T^{k}}{k z^{k}}
$$

which is obtained similarly as (2.1). Since in the equation (2.8) we have $\bar{\sigma}_{n}=0$, then the equation for finding $T_{1}, \ldots, T_{n}$ has the form

$$
\left|\begin{array}{ccccc}
s_{1}-T & 1 & 0 & \ldots & 0 \\
s_{2}-T^{2} & s_{1}-T & 2 & \ldots & 0 \\
\vdots & \vdots & \vdots & \ddots & \vdots \\
s_{n-1}-T^{n-1} & s_{n-2}-T^{n-2} & s_{n-3}-T^{n-3} & \ldots & n-1 \\
s_{n}-T^{n} & s_{n-1}-T^{n-1} & s_{n-2}-T^{n-2} & \ldots & s_{1}-T
\end{array}\right|=0 .
$$

Remark 2.1. The formula for determining the power sums $S_{k}=\sum_{i=1}^{n} T_{i}^{k}, k=$ $1, \ldots, n$, by the elementary symmetric functions $\sigma_{k}$ has the form (2.5). Now we propose the formula for determining the power sums $S_{k}=\sum_{i=1}^{n} T_{i}^{k}$, for $k=n+$ $1, n+2, \ldots, \infty$ by the elementary symmetric functions $\sigma_{k}$. This formula is as follows:

$$
S_{k}=\left|\begin{array}{cccccccc}
\sigma_{1} & 2 \sigma_{2} & \ldots & n \sigma_{n} & 0 & \ldots & 0 & 0 \\
1 & \sigma_{1} & \ldots & \sigma_{n-1} & \sigma_{n} & \ldots & 0 & 0 \\
0 & 1 & \ldots & \sigma_{n-2} & \sigma_{n-1} & \ldots & 0 & 0 \\
\vdots & \vdots & \ddots & \vdots & \vdots & \ddots & \vdots & \vdots \\
0 & 0 & \ldots & \sigma_{1} & \sigma_{2} & \ldots & \sigma_{n} & 0 \\
0 & 0 & \ldots & 1 & \sigma_{1} & \ldots & \sigma_{n-1} & \sigma_{n} \\
\vdots & \vdots & \ddots & \vdots & \vdots & \ddots & \vdots & \vdots \\
0 & 0 & \ldots & 0 & 0 & \ldots & 1 & \sigma_{1}
\end{array}\right|,
$$

where $k$ is a number of columns. Indeed, expanding the determinant along the last column we obtain Newton's identity

$$
S_{k}-\sigma_{1} S_{k-1}+\sigma_{2} S_{k-2}-\cdots+(-1)^{n} \sigma_{n} S_{k-n}=0, k>n .
$$




\section{Power sum Systems With EVEn Gaps}

In this section we consider the problem of finding a connection between odd power sums $S_{2 k-1}, k=1, \ldots, n$, and elementary symmetric functions $\sigma_{1}, \sigma_{2}, \ldots$, $\sigma_{n}$. To this end we complete the finite system of equalities (1.8) to the infinite system of equations

$$
\sum_{i=1}^{n} T_{i}^{2 k-1}=S_{2 k-1}, \quad k=1,2, \ldots, \infty .
$$

This infinite system of equations follows from the equality

$$
\sum_{i=1}^{n} \operatorname{arth} \frac{T_{i}}{z}=\sum_{k=1}^{\infty} \frac{S_{2 k-1}}{(2 k-1) z^{2 k-1}}, \quad z \in \mathbb{C},
$$

which is obtained similarly as for (2.1). Define

$$
\begin{aligned}
& R_{1}(z)=\frac{\sigma_{1} z^{n-1}+\sigma_{3} z^{n-3}+\cdots+\sigma_{n-3} z^{3}+\sigma_{n-1} z}{z^{n}+\sigma_{2} z^{n-2}+\cdots+\sigma_{n-2} z^{2}+\sigma_{n}}, \\
& R_{2}(z)=\frac{\sigma_{1} z^{n-1}+\sigma_{3} z^{n-3}+\cdots+\sigma_{n-2} z^{2}+\sigma_{n}}{z^{n}+\sigma_{2} z^{n-2}+\cdots+\sigma_{n-3} z^{3}+\sigma_{n-1} z},
\end{aligned}
$$

where $R_{1}(z)$ is for even $n$ and $R_{2}(z)$ is for odd $n$. From the formula (3.1) we have

$$
\operatorname{arth} R_{1}(z)=\sum_{k=1}^{\infty} \frac{S_{2 k-1}}{(2 k-1) z^{2 k-1}}, \quad \operatorname{arth} R_{2}(z)=\sum_{k=1}^{\infty} \frac{S_{2 k-1}}{(2 k-1) z^{2 k-1}}
$$

The form of the left-hand side of equalities (3.3) can be established, for example, by the use of the mathematical induction method.

Later on with the aid of these functional equalities we obtain the connection between the elementary symmetric functions and the odd power sums, that is, the analogs of Newton's identities. We also obtain the explicit expressions of elementary symmetric functions $\sigma_{1}, \ldots, \sigma_{n}$ in terms of odd power sums $S_{1}, S_{3}, \ldots, S_{2 n-1}$.

At first let us consider the case of the even $n$. We represent the rational function $R_{1}(z)$ in the series form, $R_{1}(z)=\sum_{k=1}^{\infty} \frac{\gamma_{2 k-1}}{z^{2 k-1}}$. Differentiating the equality

$$
\operatorname{arth} \sum_{k=1}^{\infty} \frac{\gamma_{2 k-1}}{z^{2 k-1}}=\sum_{k=1}^{\infty} \frac{S_{2 k-1}}{(2 k-1) z^{2 k-1}}
$$

with respect to $1 / z$ and comparing the coefficients with the same powers of $z$, we obtain the system of equalities that allows us to express $\gamma_{1}, \gamma_{3}, \ldots, \gamma_{2 k-1}, \ldots$ in terms of odd power sums $S_{1}, S_{3}, \ldots, S_{2 n-1}$, namely

$$
\begin{aligned}
& \gamma_{1}=S_{1} \\
& \gamma_{2 k-1}=\frac{1}{2 k-1}\left(S_{2 k-1}-\sum_{i=1}^{k-1} S_{2 k-2 i-1} \sum_{j=1}^{i} \gamma_{2 j-1} \gamma_{2 i-2 j+1}\right), \quad k=1,2, \ldots, \infty
\end{aligned}
$$


Note that the explicit formulas for $S_{k}$ can be found easily, namely

$$
S_{2 k-1}=\left|\begin{array}{ccccc}
\gamma_{1} & 3 \gamma_{3} & 5 \gamma_{5} & \ldots & (2 k-1) \gamma_{2 k-1} \\
-1 & \gamma_{1}^{2} & 2 \gamma_{1} \gamma_{3} & \ldots & \sum_{\substack{i=1 \\
k-1}}^{k-2} \gamma_{2 i-1} \gamma_{2 k-2 i-1} \\
0 & -1 & \gamma_{1}^{2} & \ldots & \sum_{i=1}^{k-1} \gamma_{2 i-1} \gamma_{2 k-2 i-3} \\
\vdots & \vdots & \vdots & \ddots & \vdots \\
0 & 0 & 0 & \ldots & 2 \gamma_{1} \gamma_{3} \\
0 & 0 & 0 & \ldots & \gamma_{1}^{2}
\end{array}\right|, \quad k=1, \ldots, n .
$$

On the other hand, the explicit expressions for $\gamma_{1}=\gamma\left(S_{1}\right), \ldots, \gamma_{2 k-1}=\gamma_{2 k-1}\left(S_{1}\right.$, $\left.\ldots, S_{2 k-1}\right)$ are more complicated, but can be found successively from this system. The dependence of $\gamma_{2 k-1}$ on the elementary symmetric functions is found from the equality

$$
R_{1}(z)=\frac{\sigma_{1} z^{n-1}+\sigma_{3} z^{n-3}+\cdots+\sigma_{n-3} z^{3}+\sigma_{n-1} z}{z^{n}+\sigma_{2} z^{n-2}+\cdots+\sigma_{n-2} z^{2}+\sigma_{n}}=\sum_{k=1}^{\infty} \frac{\gamma_{2 k-1}}{z^{2 k-1}} .
$$

From this relation we obtain the equalities

$$
\begin{aligned}
& \sum_{i=1}^{k-1} \gamma_{2 i-1} \sigma_{2 k-2 i}-\sigma_{2 k-1}=-\gamma_{2 k-1}, \quad k=1, \ldots n / 2, \\
& \sum_{i=1}^{n / 2} \gamma_{2 k-n+2 i-3} \sigma_{n-2 i+2}=-\gamma_{2 k-1}, \quad k=n / 2+1, \ldots, \infty,
\end{aligned}
$$

that connect $\sigma_{1}, \sigma_{2}, \ldots, \sigma_{n}$ and $\gamma_{1}, \gamma_{3}, \ldots$ Since from equalities (3.4) the values $\gamma_{1}, \gamma_{3}, \ldots$ are determined successively by the odd power sums, the equalities (3.5) give the connection between the elementary symmetric functions and odd power sums $S_{1}, \ldots, S_{2 n-1}$.

The equalities we obtained, namely (3.5), are analogs of Newton's formulas and express the connection between elementary symmetric functions $\sigma_{1}, \ldots, \sigma_{n}$ and odd power sums $S_{1}, \ldots, S_{2 n-1}$ in the case of even $n$.

Now let us find explicit expressions for $\sigma_{1}, \ldots, \sigma_{n}$ in terms of odd power sums $S_{1}, \ldots, S_{2 n-1}$. To this end we write the first $n$ equalities from (3.5) in the vector form

$$
A \sigma=-\gamma,
$$

where

$$
A=\left(\begin{array}{cccccccc}
-1 & 0 & 0 & 0 & \ldots & 0 & 0 & 0 \\
0 & \gamma_{1} & -1 & 0 & \ldots & 0 & 0 & 0 \\
0 & \gamma_{3} & 0 & \gamma_{1} & \ldots & 0 & 0 & 0 \\
\vdots & \vdots & \vdots & \vdots & \ddots & \vdots & \vdots & \vdots \\
0 & \gamma_{n-3} & 0 & \gamma_{n-5} & \ldots & \gamma_{1} & -1 & 0 \\
0 & \gamma_{n-1} & 0 & \gamma_{n-3} & \ldots & \gamma_{3} & 0 & \gamma_{1} \\
\vdots & \vdots & \vdots & \vdots & \ddots & \vdots & \vdots & \vdots \\
0 & \gamma_{2 n-3} & 0 & \gamma_{2 n-5} & \ldots & \gamma_{n+1} & 0 & \gamma_{n-1}
\end{array}\right), \sigma=\left(\begin{array}{c}
\sigma_{1} \\
\sigma_{2} \\
\vdots \\
\sigma_{n}
\end{array}\right), \gamma=\left(\begin{array}{c}
\gamma_{1} \\
\gamma_{3} \\
\vdots \\
\gamma_{2 n-1}
\end{array}\right)
$$


where $\gamma_{2 i-1}=\gamma_{2 i-1}\left(S_{1}, S_{3}, \ldots, S_{2 n-1}\right)$. Observe that $\operatorname{det} A=(-1)^{n / 2} \tilde{\Delta}_{1, n / 2}$. Suppose that $\tilde{\Delta}_{1, n / 2}$ is nonzero, then from (3.6) we obtain the equality

$$
\sigma=-A^{-1} \gamma
$$

Suppose that odd power sums are given numbers $s_{1}, s_{3}, \ldots, s_{2 n-1}$. Then finding unknowns $T_{1}, \ldots, T_{n}$ in the system (1.8) is reduced to the determination of $n$ roots of the polynomial $P_{n}(t)$ of the form $P_{n}(t)=t^{n}-\sigma_{1} t^{n-1}+\cdots-\sigma_{n-1} t+\sigma_{n}$ or, equivalently, solving the equation

$$
\begin{array}{|cccccccc}
\gamma_{1} & 1 & 0 & 0 & 0 & \ldots & 0 & 0 \\
\gamma_{3} & 0 & \gamma_{1} & 1 & 0 & \ldots & 0 & 0 \\
\gamma_{5} & 0 & \gamma_{3} & 0 & \gamma_{1} & \ldots & 0 & 0 \\
\vdots & \vdots & \vdots & \vdots & \vdots & \ddots & \vdots & \vdots \\
\gamma_{n-1} & 0 & \gamma_{n-3} & 0 & \gamma_{n-5} & \ldots & 1 & 0 \\
\gamma_{n+1} & 0 & \gamma_{n-1} & 0 & \gamma_{n-3} & \ldots & 0 & \gamma_{1} \\
\vdots & \vdots & \vdots & \vdots & \vdots & \ddots & \vdots & \vdots \\
\gamma_{2 n-1} & 0 & \gamma_{2 n-3} & 0 & \gamma_{2 n-5} & \ldots & 0 & \gamma_{n-1} \\
t^{n} & t^{n-1} & t^{n-2} & t^{n-3} & t^{n-4} & \ldots & t & 1
\end{array}
$$

Now we consider the case of odd $n$. As in the previous case we represent the rational function $R_{2}(z)$ in the form

$$
R_{2}(z)=\frac{\sigma_{1} z^{n-1}+\sigma_{3} z^{n-3}+\cdots+\sigma_{n-2} z^{2}+\sigma_{n}}{z^{n}+\sigma_{2} z^{n-2}+\cdots+\sigma_{n-3} z^{3}+\sigma_{n-1} z}=\sum_{k=1}^{\infty} \frac{\gamma_{2 k-1}}{z^{2 k-1}} .
$$

As before, $\gamma_{2 k-1}, k=1,2, \ldots$, can be expressed from the equality (3.4) in terms of $S_{1}, \ldots, S_{2 n-1}$. From the formula 3.9 we can formulate the system of linear equations

$$
\begin{aligned}
& \sum_{i=1}^{k-1} \gamma_{2 i-1} \sigma_{2 k-2 i}-\sigma_{2 k-1}=-\gamma_{2 k-1}, \quad k=1, \ldots,(n+1) / 2, \\
& \sum_{i=1}^{(n-1) / 2} \gamma_{2 k+2 i-n-2} \sigma_{n-2 i+1}=-\gamma_{2 k-1}, \quad k=(n+3) / 2, \ldots, \infty
\end{aligned}
$$

for expressing of $\sigma_{1}, \ldots, \sigma_{n}$ via $\gamma_{1}, \ldots, \gamma_{n}$. To this end we write the first $n$ equalities from (3.10) in the vector form

$$
A_{1} \sigma=-\gamma
$$

where

$$
A_{1}=\left(\begin{array}{ccccccc}
-1 & 0 & 0 & 0 & \ldots & 0 & 0 \\
0 & \gamma_{1} & -1 & 0 & \ldots & 0 & 0 \\
0 & \gamma_{3} & 0 & \gamma_{1} & \ldots & 0 & 0 \\
\vdots & \vdots & \vdots & \vdots & \ddots & \vdots & \vdots \\
0 & \gamma_{n-4} & 0 & \gamma_{n-6} & \ldots & 0 & 0 \\
0 & \gamma_{n-2} & 0 & \gamma_{n-4} & \ldots & \gamma_{1} & -1 \\
\vdots & \vdots & \vdots & \vdots & \ddots & \vdots & \vdots \\
0 & \gamma_{2 n-3} & 0 & \gamma_{2 n-5} & \ldots & \gamma_{n} & 0
\end{array}\right), \gamma=\left(\begin{array}{c}
\gamma_{1} \\
\gamma_{3} \\
\vdots \\
\gamma_{2 n-1}
\end{array}\right), \sigma=\left(\begin{array}{c}
\sigma_{1} \\
\sigma_{2} \\
\vdots \\
\sigma_{n}
\end{array}\right)
$$

This system has the unique solution if Hankel's determinant $\widetilde{\Delta}_{3, \frac{n-1}{2}} \neq 0$. 
The connection of elementary symmetric functions with the odd sums is given by the equality

$$
\sigma=-A_{1}^{-1} \gamma
$$

Solving the system (1.8) for odd $n$ and given numbers $s_{1}, \ldots, s_{2 n-1}$, is reduced to finding the roots of the polynomial $Q_{n}(t)=t^{n}-\sigma_{1} t^{n-1}+\cdots+\sigma_{n-1} t-\sigma_{n}$ or, equivalently, solving the equation

$$
\left|\begin{array}{ccccccc}
\gamma_{1} & 1 & 0 & 0 & \ldots & 0 & 0 \\
\gamma_{3} & 0 & \gamma_{1} & 1 & \ldots & 0 & 0 \\
\gamma_{5} & 0 & \gamma_{3} & 0 & \ldots & 0 & 0 \\
\vdots & \vdots & \vdots & \vdots & \ddots & \vdots & \vdots \\
\gamma_{n} & 0 & \gamma_{n-2} & 0 & \ldots & \gamma_{1} & 1 \\
\vdots & \vdots & \vdots & \vdots & \ddots & \vdots & \vdots \\
\gamma_{2 n-1} & 0 & \gamma_{2 n-3} & 0 & \ldots & \gamma_{n} & 0 \\
t^{n} & t^{n-1} & t^{n-2} & t^{n-3} & \ldots & t & 1
\end{array}\right|=0 .
$$

Summarizing, we proved

Theorem 3.1. The equality (3.5) for even $n$ and the equality (3.10) for odd $n$ express the connection between elementary symmetric functions $\sigma_{1}, \ldots, \sigma_{n}$ and odd power sums $S_{1}, \ldots, S_{2 n-1}, \ldots$

This theorem is an analog of Newton's formulas.

Example 3.1. Let $n=4$. Let us find the expression of the elementary symmetric functions $\sigma_{1}, \sigma_{2}, \sigma_{3}, \sigma_{4}$ by odd power sums $S_{1}, S_{3}, S_{5}, S_{7}$. Using (3.4), we obtain

$$
\begin{aligned}
& \gamma_{1}=S_{1}, \quad \gamma_{3}=\frac{1}{3}\left(-S_{1}^{3}+S_{3}\right), \quad \gamma_{5}=\frac{1}{15}\left(2 S_{1}^{5}-5 S_{1}^{2} S_{3}+3 S_{5}\right), \\
& \gamma_{7}=\frac{1}{315}\left(-17 S_{1}^{7}+70 S_{1}^{4} S_{3}-35 S_{1} S_{3}^{2}-63 S_{1}^{2} S_{5}+45 S_{7}\right) .
\end{aligned}
$$

System (3.6) has the form

$$
\sigma_{1}=\gamma_{1}, \sigma_{3}=\gamma_{3}+\gamma_{1} \sigma_{2}, \quad 0=\gamma_{5}+\gamma_{1} \sigma_{4}+\gamma_{3} \sigma_{2}, \quad 0=\gamma_{7}+\gamma_{3} \sigma_{4}+\gamma_{5} \sigma_{2} .
$$

Suppose that $\Delta=\tilde{\Delta}_{1,2}=\left|\begin{array}{cc}\gamma_{1} & \gamma_{3} \\ \gamma_{3} & \gamma_{5}\end{array}\right| \neq 0$, where $\tilde{\Delta}_{1,2}=9 S_{1} S_{5}-5 S_{1}^{3} S_{3}+S_{1}^{6}-5 S_{3}^{2}$.

Then taking into account equalities (3.14) we have

$$
\begin{aligned}
& \sigma_{1}=S_{1}, \quad \sigma_{2}=\frac{3\left(-15 S_{1} S_{7}+14 S_{1}^{3} S_{5}-7 S_{1}^{5} S_{3}+S_{1}^{8}+7 S_{3} S_{5}\right)}{7 \Delta}, \\
& \sigma_{3}=\frac{126 S_{1} S_{3} S_{5}-135 S_{1}^{2} S_{7}+63 S_{1}^{4} S_{5}-21 S_{1}^{6} S_{3}+2 S_{1}^{9}-35 S_{3}^{3}}{21 \Delta}, \\
& \sigma_{4}=\frac{-175 S_{1} S_{3}^{3}+315 S_{1}^{2} S_{3} S_{5}-225 S_{1}^{3} S_{7}+63 S_{1}^{5} S_{5}-15 S_{1}^{7} S_{3}+S_{1}^{10}+225 S_{3} S_{7}-189 S_{5}^{2}}{105 \Delta} .
\end{aligned}
$$


Considering the system (1.8) with given $s_{1}, s_{3}, s_{5}, s_{7}$ with respect to unknown $T_{1}$, $T_{2}, T_{3}, T_{4}$, we have the equation of the form

$$
t^{4}-\sigma_{1} t^{3}+\sigma_{2} t^{2}-\sigma_{3} t+\sigma_{4}=0
$$

for finding of $T_{1}, T_{2}, T_{3}, T_{4}$, where $\sigma_{1}, \sigma_{2}, \sigma_{3}, \sigma_{4}$ are defined by equalities (3.15). This equation may also be written in the form

$$
\left|\begin{array}{ccccc}
\gamma_{1} & 1 & 0 & 0 & 0 \\
\gamma_{3} & 0 & \gamma_{1} & 1 & 0 \\
\gamma_{5} & 0 & \gamma_{3} & 0 & \gamma_{1} \\
\gamma_{7} & 0 & \gamma_{5} & 0 & \gamma_{3} \\
t^{4} & t^{3} & t^{2} & t & 1
\end{array}\right|=0
$$

Example 3.2. Let us find the nodes of quadrature formulas with even gaps of Chebyshev and Mehler types in the case $n=4$. The system of equations for determination of nodes of the quadrature formula has the form

$$
T_{1}^{2 k-1}+T_{2}^{2 k-1}+T_{3}^{2 k-1}+T_{4}^{2 k-1}+T_{5}^{2 k-1}=s_{2 k-1}, \quad k=1, \ldots, 4 .
$$

Let us find nodes of quadrature formula with even gaps of Chebyshev type for $a=1, b=2$. In this case odd power sums are known numbers, namely

$$
s_{2 k-1}=\frac{n\left(2^{2 k}-1\right)}{2 k}, \quad k=1, \ldots, n .
$$

For $n=4$ we have $s_{1}=6, s_{3}=15, s_{5}=42, s_{7}=127.5$. From (3.15) we obtain $\sigma_{1}=6, \sigma_{2}=327693 / 24577, \sigma_{3}=319499 / 24577, \sigma_{4}=1152351 / 245770$. Then the nodes are the roots of the equation $(3.16)$ and we have $T_{1} \approx 1.1020151, T_{2} \approx$ $1.4085047, T_{3} \approx 1.5917667, T_{4} \approx 1.8977134$.

In the case of quadrature formula with even gaps of Mehler type for $a=0, b=1$ we have

$$
s_{2 k-1}=\frac{2 n}{\pi} \cdot \frac{(2 k-2) ! !}{(2 k-1) ! !}, \quad k=1, \ldots, n .
$$

For $n=4$ we have $s_{1}=8 / \pi, s_{3}=16 / 3 \pi, s_{5}=64 / 15 \pi, s_{7}=128 / 35 \pi$. From (3.15) we get $\sigma_{1} \approx 2.546479, \sigma_{2} \approx 2.243998 \sigma_{3} \approx 0.775916, \sigma_{4} \approx 0.079528$. Then the nodes are the roots of the equation $(3.16)$ and on equal $T_{1} \approx 0.173803284, T_{2} \approx$ $0.562862793, T_{3} \approx 0.828011294, T_{4} \approx 0.981801718$.

Using obtained nodes for quadrature formulas with even gaps of Chebyshev and Mehler types, for example, for $f(t)=\sin t$, we obtain

$$
\int_{1}^{2} \sin t d t \approx 0.956449, \quad \int_{0}^{1} \frac{\sin t}{\sqrt{1-t^{2}}} d t \approx 0.89324374 .
$$

Consider the cases when Hankel determinants $\tilde{\Delta}_{1, n / 2}$ and $\tilde{\Delta}_{1,(n-1) / 2}$ are equal to zero. In these cases the system (1.8) for even $n$ and odd $n$ has the infinite set of solutions if the conditions $\operatorname{rank}\left(\Gamma_{1, n / 2}^{2}\right)=\operatorname{rank}\left(\Gamma_{1, n / 2+1}^{2}\right)$, and $\operatorname{rank}\left(\Gamma_{1,(n-1) / 2}^{2}\right)=$ $\operatorname{rank}\left(\Gamma_{1,(n+1) / 2}^{2}\right)$ are fulfilled, respectively. In the case of even $n$, if $\tilde{\Delta}_{1, n / 2}=0$ and the condition $\operatorname{rank}\left(\Gamma_{1, n / 2}^{2}\right)=\operatorname{rank}\left(\Gamma_{1,1+n / 2}^{2}\right)$ does not hold, then the system (1.8) does not have a solution. Likewise, in the case of odd $n$, if $\tilde{\Delta}_{1,(n-1) / 2}=0$ and the condition $\operatorname{rank}\left(\Gamma_{1,(n-1) / 2}^{2}\right)=\operatorname{rank}\left(\Gamma_{1,(n+1) / 2}^{2}\right)$ is not satisfied, then the system (1.8) has no solution. We illustrate those results by the following example. 
Example 3.3. Put $n=4$. Initially, suppose that $\tilde{\Delta}_{1,2} \neq 0$. It means that $s_{1} \neq 0$ and $s_{5} \neq\left(-s_{1}^{6}+5 s_{1}^{3} s_{3}+5 s_{3}^{2}\right) /\left(9 s_{1}\right)$, or $s_{1}=0, s_{3} \neq 0$. Then the system

$$
T_{1}^{2 k-1}+T_{2}^{2 k-1}+T_{3}^{2 k-1}+T_{4}^{2 k-1}=s_{2 k-1}, \quad k=1, \ldots, 4,
$$

has a unique (up to permutation) solution $T_{1}, T_{2}, T_{3}, T_{4}$.

Now let $\tilde{\Delta}_{1,2}=0$ and $\operatorname{rank}\left(\begin{array}{ll}\gamma_{1} & \gamma_{3} \\ \gamma_{3} & \gamma_{5}\end{array}\right)=\operatorname{rank}\left(\begin{array}{lll}\gamma_{1} & \gamma_{3} & \gamma_{5} \\ \gamma_{3} & \gamma_{5} & \gamma_{7}\end{array}\right)$. Then the system (3.17) has the infinite set of solutions only if $s_{1} \neq 0, s_{5}=\left(-s_{1}^{6}+5 s_{1}^{3} s_{3}+5 s_{3}^{2}\right) /\left(9 s_{1}\right)$, $s_{7}=\left(-s_{1}^{9}+21 s_{1}^{3} s_{3}^{2}+7 s_{3}^{3}\right) /\left(27 s_{1}^{2}\right)$ or if $s_{1}=s_{3}=s_{5}=s_{7}=0$.

Put $s_{1}=1, s_{3}=1$. Then $s_{5}=s_{7}=1$ and the system (3.17) has the infinite number of solutions $T_{1}=-T_{2}, T_{3}=0$ and $T_{4}=1$.

Finally, suppose that $\tilde{\Delta}_{1,2}=0$ and $\operatorname{rank}\left(\begin{array}{ll}\gamma_{1} & \gamma_{3} \\ \gamma_{3} & \gamma_{5}\end{array}\right) \neq \operatorname{rank}\left(\begin{array}{lll}\gamma_{1} & \gamma_{3} & \gamma_{5} \\ \gamma_{3} & \gamma_{5} & \gamma_{7}\end{array}\right)$. Then the system (3.17) has no solution if $s_{1} \neq 0, s_{5}=\left(-s_{1}^{6}+5 s_{1}^{3} s_{3}+5 s_{3}^{2}\right)\left(9 s_{1}\right), s_{7} \neq$ $\left(-s_{1}^{9}+21 s_{1}^{3} s_{3}^{2}+7 s_{3}^{3}\right)\left(27 s_{1}^{2}\right)$ or if $s_{1}=s_{3}=0, s_{5} \neq 0$.

\section{Solving ALmost POWER SUM SYSTEMS With EVEN GAPS}

Let us consider the almost polynomial system (1.2) with even gaps and with an arbitrary number of positive and negative summands. Using a similar method to the one used in the previous section, we complete the finite system of equalities (1.2) to the infinite system of equations

$$
-\sum_{i=1}^{m} T_{i}^{2 k-1}+\sum_{i=m+1}^{n-1} T_{i}^{2 k-1}=C_{2 k-1}, \quad k=1,2, \ldots, \infty,
$$

where $C_{2 k-1}=-f_{2 k-1}\left(\varphi\left(T_{1}, \ldots, T_{n}\right)\right)$ for $1 \leq k \leq n$, and $C_{2 k-1}$ is unspecified for $k>n ; C_{2 k-1}$ for $k>n$ is introduced to complete the system in (1.2) to an infinite system, and they are not used in solving (1.2). Similarly to (3.1) we have

$$
-\sum_{i=1}^{m} \operatorname{arth} \frac{T_{i}}{z}+\sum_{i=m+1}^{n-1} \operatorname{arth} \frac{T_{i}}{z}=\sum_{k=1}^{\infty} \frac{C_{2 k-1}}{(2 k-1) z^{2 k-1}} .
$$

We want to use methods in previous sections to (4.2), but we need to complement it, because now we have summands of different signs. In this case, the sum in the left-hand side of (4.2) cannot be expressed via $\sigma_{k}$ 's in a similar way as for functions $R_{1}, R_{2}$ given in (3.2). We want to obtain analogous formulas to (3.2) in this case. Therefore we introduce new symmetric functions $\widetilde{\sigma}_{k}$ and $\widehat{\sigma}_{k}$, in a similar way as in (1.10), putting $\widetilde{\sigma}_{1}=T_{1}+\cdots+T_{m}, \ldots, \widetilde{\sigma}_{m}=T_{1} T_{2} \ldots T_{m}$, and $\widehat{\sigma}_{1}=T_{m+1}+\cdots+T_{n-1}$, $\ldots, \widehat{\sigma}_{n-m-1}=T_{m+1} T_{m+2} \ldots T_{n-1}$. Similar to (3.2) we introduce

$$
\widehat{R}_{1}(z)=\frac{\widehat{\sigma}_{1} z^{n-m-2}+\cdots+\widehat{\sigma}_{n-m-2} z}{z^{n-m-1}+\cdots+\widehat{\sigma}_{n-m-1}}, \quad \widehat{R}_{2}(z)=\frac{\widehat{\sigma}_{1} z^{n-m-2}+\cdots+\widehat{\sigma}_{n-m-1}}{z^{n-m-1}+\cdots+\widehat{\sigma}_{n-m-2} z},
$$

where $\widetilde{R}_{1}(z)$ is for even $m, \widetilde{R}_{2}(z)$ is for odd $m ; \widehat{R}_{1}(z)$ is for even $n-m-1$, and $\widehat{R}_{2}(z)$ is for odd $n-m-1$.

Then, using similar arguments as before, we obtain

$$
-\sum_{i=1}^{m} \operatorname{arth} \frac{T_{i}}{z}+\sum_{i=m+1}^{n-1} \operatorname{arth} \frac{T_{i}}{z}=-\operatorname{arth} \widetilde{R}_{1}(z)+\operatorname{arth} \widehat{R}_{1}(z)=\operatorname{arth} \frac{-\widetilde{R}_{1}(z)+\widehat{R}_{1}(z)}{1-\widetilde{R}_{1}(z) \widehat{R}_{1}(z)}
$$


when $m$ and $n-m-1$ are even,

$$
-\sum_{i=1}^{m} \operatorname{arth} \frac{T_{i}}{z}+\sum_{i=m+1}^{n-1} \operatorname{arth} \frac{T_{i}}{z}=\operatorname{arth} \frac{-\widetilde{R}_{1}(z)+\widehat{R}_{2}(z)}{1-\widetilde{R}_{1}(z) \widehat{R}_{2}(z)}
$$

when $m$ is even and $n-m-1$ is odd,

$$
-\sum_{i=1}^{m} \operatorname{arth} \frac{T_{i}}{z}+\sum_{i=m+1}^{n-1} \operatorname{arth} \frac{T_{i}}{z}=\operatorname{arth} \frac{-\widetilde{R}_{2}(z)+\widehat{R}_{1}(z)}{1-\widetilde{R}_{2}(z) \widehat{R}_{1}(z)}
$$

when $m$ is odd and $n-m-1$ is even,

$$
-\sum_{i=1}^{m} \operatorname{arth} \frac{T_{i}}{z}+\sum_{i=m+1}^{n-1} \operatorname{arth} \frac{T_{i}}{z}=\operatorname{arth} \frac{-\widetilde{R}_{2}(z)+\widehat{R}_{2}(z)}{1-\widetilde{R}_{2}(z) \widehat{R}_{2}(z)}
$$

when $m$ and $n-m-1$ are odd. For example, if $m=1$ and $n=4$, the above means that

$$
\begin{aligned}
-\operatorname{arth} \frac{T_{1}}{z}+\operatorname{arth} \frac{T_{2}}{z}+\operatorname{arth} \frac{T_{3}}{z} & =\frac{\left(-\widetilde{\sigma}_{1}+\widehat{\sigma}_{1}\right) z^{2}-\widetilde{\sigma}_{1} \widehat{\sigma}_{2}}{z^{3}+\left(-\widehat{\sigma}_{1} \widetilde{\sigma}_{1}+\widehat{\sigma}_{2}\right) z} \\
& =\frac{\left(-T_{1}+T_{2}+T_{3}\right) z^{2}-T_{1} T_{2} T_{3}}{z^{3}+\left(-T_{2} T_{1}-T_{3} T_{1}+T_{2} T_{3}\right) z} .
\end{aligned}
$$

We can express those equalities in the simpler form

$$
-\sum_{i=1}^{m} \operatorname{arth} \frac{T_{i}}{z}+\sum_{i=m+1}^{n-1} \operatorname{arth} \frac{T_{i}}{z}=\left\{\begin{array}{l}
\operatorname{arth} \bar{R}_{1}(z) \text { for } n \text { even, } \\
\operatorname{arth} \bar{R}_{2}(z) \text { for } n \text { odd }
\end{array}\right.
$$

where

$$
\begin{gathered}
\bar{R}_{1}(z)=\frac{b_{1} z^{n-2}+b_{3} z^{n-4}+\cdots+b_{n-3} z^{2}+b_{n-1}}{z^{n-1}+b_{2} z^{n-3}+\cdots+b_{n-4} z^{3}+b_{n-2} z}, \\
\bar{R}_{2}(z)=\frac{b_{1} z^{n-2}+b_{3} z^{n-4}+\cdots+b_{n-4} z^{3}+b_{n-2} z}{z^{n-1}+b_{2} z^{n-3}+\cdots+b_{n-3} z^{2}+b_{n-1}},
\end{gathered}
$$

and $b_{j}$ are some nonsymmetric functions of $\widetilde{\sigma}_{k}$ and $\widehat{\sigma}_{k}$. Thus we obtained similar expressions as in (3.2). Now we can proceed with further analysis.

At first we consider the case of even $n$. The left-hand side of this equation has an odd number of terms. Then in analogy to the first equation of (3.3), the equation (4.2) has the form $\operatorname{arth} \bar{R}_{1}=\sum_{k=1}^{\infty} \frac{C_{2 k-1}}{(2 k-1) z^{2 k-1}}$, where the rational function $\bar{R}_{1}(z)$ has the following series expansion:

$$
\bar{R}_{1}(z)=\frac{b_{1} z^{n-2}+b_{3} z^{n-4}+\cdots+b_{n-3} z^{2}+b_{n-1}}{z^{n-1}+b_{2} z^{n-3}+\cdots+b_{n-4} z^{3}+b_{n-2} z}=\sum_{k=1}^{\infty} \frac{\gamma_{2 k-1}}{z^{2 k-1}} .
$$

Here, for each $k \in\{1, \ldots, n\}, \gamma_{2 k-1}$ is a function in $\varphi\left(T_{1}, \ldots, T_{n}\right)$ and defined by the equality

$$
\operatorname{arth} \sum_{k=1}^{\infty} \frac{\gamma_{2 k-1}}{z^{2 k-1}}=\sum_{k=1}^{\infty} \frac{C_{2 k-1}}{(2 k-1) z^{2 k-1}} .
$$

As before we obtain the expressions for $\gamma_{2 k-1}, k=1 \ldots n$, namely

$$
\gamma_{1}=C_{1}, \quad \gamma_{2 k-1}=\frac{1}{2 k-1}\left(C_{2 k-1}-\sum_{i=1}^{k-1} C_{2 k-2 i-1} \sum_{j=1}^{i} \gamma_{2 j-1} \gamma_{2 i-2 j+1}\right)
$$


From equality (4.5) it follows that

$$
\begin{aligned}
b_{n-2} \gamma_{3}+b_{n-4} \gamma_{5}+\cdots+b_{2} \gamma_{n-1}+\gamma_{n+1} & =0, \\
b_{n-2} \gamma_{5}+b_{n-4} \gamma_{7}+\cdots+b_{2} \gamma_{n+1}+\gamma_{n+3} & =0, \\
& \vdots \\
b_{n-2} \gamma_{n+1}+b_{n-4} \gamma_{n+3}+\cdots+b_{2} \gamma_{2 n-3}+\gamma_{2 n-1} & =0 .
\end{aligned}
$$

This system of equations has a nonzero solution, namely $1, b_{2}, \ldots, b_{n-2}$, hence we obtain

$$
\widetilde{\Delta}_{3, n / 2}=0,
$$

where $\widetilde{\Delta}_{i, k}$ was defined in (1.11). Let $T_{1}^{0}, \ldots, T_{n}^{0}$ be some solutions of the system (4.1). Then (4.7) is an equation that allows us to find the values of the function $\varphi\left(T_{1}^{0}, \ldots, T_{n}^{0}\right)$. Suppose (4.7) has a solution. We denote by $\bar{\varphi}=\varphi\left(T_{1}^{0}, \ldots, T_{n}^{0}\right)$ one of the solutions of this equation.

In the case of odd $n$ in analogy to (4.5) changing $\bar{R}_{2}(z)$ to $\bar{R}_{1}(z)$ we obtain a similar equation for finding the value $\bar{\varphi}=\varphi\left(T_{1}^{0}, \ldots, T_{n}^{0}\right)$, namely

$$
\widetilde{\Delta}_{1,(n+1) / 2}=0 \text {. }
$$

Again, let $T_{1}^{0}, \ldots, T_{n}^{0}$ be some solutions of system (4.1), and suppose the equation (4.7) has a solution. We denote by $\bar{\varphi}=\varphi\left(T_{1}^{0}, \ldots, T_{n}^{0}\right)$ one of the solutions of this equation.

Now we will find two functions of one variable, the roots of which are unknown values $T_{1}, \ldots, T_{m}$, and $T_{m+1}, T_{m+2}, \ldots, T_{n-1}$. Let $\bar{\varphi}$ be one of the roots of equation (4.7) or (4.8). We will find the equations for $T_{m+1}, \ldots, T_{n-1}$. The equation (4.1) is symmetric with respect to the variables $T_{m+1}, \ldots, T_{n-1}$. Therefore, we denote by $T$ any of those variables. Put $T=T_{n-1}$. We rewrite the system (4.1) in the form

$$
-\sum_{i=1}^{m} T_{i}^{2 k-1}+\sum_{i=m+1}^{n-2} T_{i}^{2 k-1}=C_{2 k-1}, \quad k=1, \ldots, n,
$$

and define $\gamma_{k}$ by the values given by (4.6), where $C_{2 k-1}$ denotes the new righthand side values, that is, $C_{2 k-1}=-f_{2 k-1}(\bar{\varphi})-T^{2 k-1}$ (for simplicity of recurrent formulas we use the same notations in all steps).

Equations for $T_{m+1}, \ldots, T_{n-1}$ can be found in a similar way to obtaining (4.7) and (4.8), and have the form:

$$
\begin{gathered}
\widetilde{\Delta}_{1, n / 2}=0 \quad \text { for even } n, \\
\widetilde{\Delta}_{3,(n-1) / 2}=0 \quad \text { for odd } n, n \geq 3 .
\end{gathered}
$$

Let us denote by $T_{m+1}, \ldots T_{n-1}$ the roots of equations (4.9) or (4.10).

Now we proceed to finding the values of the variables $T_{1}, \ldots, T_{m}$, again we denote by $T$ one of them; for simplicity of our notation let $T=T_{m}$. Then the system for finding the unknowns $T_{1}, \ldots, T_{m}$ is of the form $-\sum_{i=1}^{m-1} T_{i}^{2 k-1}=C_{2 k-1}, \gamma_{2 k-1}$ being defined by the values given by (4.6), where $C_{2 k-1}=-\sum_{i=m+1}^{n-1} T_{i}^{2 k-1} f_{2 k-1}(\bar{\varphi})+$ $T^{2 k-1}$. Therefore the equations for finding $T_{1}, \ldots, T_{m}$ have the form

$$
\widetilde{\Delta}_{3, m / 2}=0 \text { for even } m,
$$

$$
\widetilde{\Delta}_{1,(m+1) / 2}=0 \text { for odd } m \text {. }
$$


This way for all values of $\bar{\varphi}$ we found the equations for $T_{1}, \ldots, T_{n-1}$. The unknown value of $T_{n}$ can be found from

$$
\varphi\left(T_{1}, T_{2}, \ldots, T_{n-1}, T_{n}\right)=\bar{\varphi}
$$

The equality (3.5) for even $n$ and the equality (3.10) for odd $n$ express the connection between elementary symmetric functions $\sigma_{1}, \ldots, \sigma_{n}$ and odd power sums $S_{1}, \ldots, S_{2 n-1}$. Summarizing, we proved

Theorem 4.1. If the equation (4.13) has a solution $T_{n}$ and the equation (4.7) (or (4.8) ) has a solution $\varphi$, then solving the system (1.2) is reduced to the successive search of the roots of four functions of one variable:

- equations (4.7) or (4.8) for finding $\bar{\varphi}$;

- polynomial equations (4.9) or (4.10) for $T_{m+1}, \ldots, T_{n-1}$;

- polynomial equations (4.11) or (4.12) for $T_{1}, \ldots, T_{m}$;

- equation (4.13) for $T_{n}$.

Note that the equation (4.13) has a solution $T_{n}$ for any $\bar{\varphi}$ if $\varphi$ is continuous, and for all fixed $T_{1}, \ldots, T_{n-1}$ its image (w.r.t. $T_{n}$ ) is the whole real line $\mathbb{R}$. For example, the equation (4.13) has the unique solution continuously differentiable with respect to $T_{n}$ if the function $\varphi\left(T_{1}, T_{2}, \ldots, T_{n-1}, T_{n}\right)$ is continuously differentiable with respect to $T_{n}$ and $\left|\frac{\partial \varphi\left(T_{1}, T_{2}, \ldots, T_{n-1}, T_{n}\right)}{\partial T_{n}}\right| \geq a>0$ for certain constant $a$.

Example 4.1. Let us consider the time-optimal control problem for the system

$$
\dot{x}_{1}=-x_{1}+u, \quad \dot{x}_{2}=-3 x_{2}+u, \quad \dot{x}_{3}=-5 x_{3}+u, \quad|u| \leq 1,
$$

from the initial point $\left(x_{1}^{0}, x_{2}^{0}, x_{3}^{0}\right)$ to the origin. This problem is equivalent to min-problem [6, 7]: we need to find the minimal value $\theta$ and a function $u(t)$ such that

$$
\int_{0}^{\theta} e^{t} u(t) d t=-x_{1}^{0}, \int_{0}^{\theta} e^{3 t} u(t) d t=-x_{2}^{0}, \quad \int_{0}^{\theta} e^{5 t} u(t) d t=-x_{3}^{0} .
$$

The control $u(t)$ is a piecewise constant function and takes the values \pm 1 , and $\theta$ is the optimal time. We denote by $T_{1}, T_{2}$ points of discontinuity (the switching moments) of $u(t)$. Put $\widetilde{T}_{1}=e^{T_{1}}, \widetilde{T}_{2}=e^{T_{2}}, \widetilde{T}_{3}=e^{\theta}$. For finding $\widetilde{T}_{1}, \widetilde{T}_{2}, \widetilde{T}_{3}$ we need to solve two systems of equations,

$$
\left\{\begin{aligned}
&-\widetilde{T}_{1}+\widetilde{T}_{2}-0.5 \widetilde{T}_{3}=0.5\left(-1+\widetilde{u} x_{1}^{0}\right), \\
&-\widetilde{T}_{1}^{3}+\widetilde{T}_{2}^{3}-0.5 \widetilde{T}_{3}^{3}=0.5\left(-1+3 \widetilde{u} x_{2}^{0}\right) \\
&-\widetilde{T}_{1}^{5}+\widetilde{T}_{2}^{5}-0.5 \widetilde{T}_{3}^{5}=0.5\left(-1+5 \widetilde{u} x_{3}^{0}\right),
\end{aligned}\right.
$$

for $\widetilde{u}=+1$ and $\widetilde{u}=-1$, where $\widetilde{u}$ the value of function $u(t)$ on the interval $\left(\widetilde{T}_{2}, \widetilde{T}_{3}\right)$. Since $\theta$ is the optimal time we find unknown $\widetilde{T}_{3}=e^{T_{3}}$ as the maximal real root (see [6, 15]) of two equations for $\widetilde{u}= \pm 1$ of the form $\left|\begin{array}{ll}\gamma_{1} & \gamma_{3} \\ \gamma_{3} & \gamma_{5}\end{array}\right|=0$, where $\gamma_{1}=C_{1}, \quad \gamma_{3}=$ $\left(C_{3}-C_{1} \gamma_{1}^{2}\right) / 3, \quad \gamma_{5}=\left(C_{5}-C_{3} \gamma_{1}^{2}-2 C_{1} \gamma_{1} \gamma_{3}\right) / 5, C_{1}=0.5\left(\widetilde{T}_{3}-1+\widetilde{u} x_{1}^{0}\right), \quad C_{3}=$ $0.5\left(\widetilde{T}_{3}^{3}-1+3 \widetilde{u} x_{2}^{0}\right), \quad C_{5}=0.5\left(\widetilde{T}_{3}^{5}-1+5 \widetilde{u} x_{3}^{0}\right)$. 
For example, let $(1,2,3)$ be the initial point, then $\widetilde{T}_{3}$ is the maximal real root of the equations

$$
\widetilde{T}_{3}^{6}-4 \widetilde{T}_{3}^{5}-4 \widetilde{T}_{3}^{4}+28 \widetilde{T}_{3}^{3}-\left(40 \widetilde{T}_{3}^{2} / 3\right)-\left(272 \widetilde{T}_{3} / 15\right)-(368 / 45)=0 \quad \text { for } \widetilde{u}=-1,
$$

or

$$
\widetilde{T}_{3}^{6}-20 \widetilde{T}_{3}^{3}+\left(224 \widetilde{T}_{3} / 5\right)-(400 / 9)=0 \quad \text { for } \widetilde{u}=+1 .
$$

Solving these equations we obtain $\widetilde{T}_{3} \approx 2.854297$ and $\widetilde{u}=-1$. Now we find $\widetilde{T}_{2}$ as the maximal real root of the equation $\bar{\gamma}_{3}=0$, where $\bar{\gamma}_{3}=\frac{1}{3}\left(\bar{C}_{3}-\bar{C}_{1}^{3}\right), \bar{C}_{1}=$ $C_{1}-\widetilde{T}_{2}, \bar{C}_{3}=C_{3}-\widetilde{T}_{2}^{3}$. We obtain $\widetilde{T}_{2}^{2}-\widetilde{T}_{2} \cdot 0.427148-6.281234=0$, therefore $\widetilde{T}_{2} \approx 2.728897$. From the first equation of the system (4.15) we have $\widetilde{T}_{1} \approx 2.301748$.

Since $T_{1}=\ln \widetilde{T}_{1}, T_{2}=\ln \widetilde{T}_{2}, \theta=\ln \widetilde{T}_{3}$ then finally we obtain the values of the optimal time $\theta$ and the switching moments $T_{1}, T_{2}$, namely

$$
T_{1} \approx 0.833669, \quad T_{2} \approx 1.003897, \quad \theta \approx 1.048826, \widetilde{u}=-1 .
$$

Example 4.2. Let us consider the system of almost polynomial nonlinear equations,

$$
\left\{\begin{array}{l}
-T_{1}+T_{1}^{2}-T_{2}+T_{3}+2 T_{1} T_{4}+T_{4}^{2}-4=0, \\
-T_{1}^{3}-T_{2}^{3}+T_{3}^{3}+3 \sin \frac{\pi\left(T_{1}+T_{4}\right)}{2}+6=0, \\
-T_{1}^{5}-T_{2}^{5}+T_{3}^{5}-2 \cos \pi\left(T_{1}+T_{4}\right)+32=0 \\
-T_{1}^{7}-T_{2}^{7}+T_{3}^{7}+126=0 .
\end{array}\right.
$$

This is the system of the form (1.2), since the first equation may be written as $-T_{1}-T_{2}+T_{3}+\left(T_{1}+T_{4}\right)^{2}-4=0$. Therefore we have $\varphi\left(T_{1}, T_{2}, T_{3}, T_{4}\right)=T_{1}+$ $T_{4}, f_{1}(\varphi)=\varphi^{2}-4, f_{2}(\varphi)=3 \sin \frac{\pi \varphi}{2}+6, f_{3}(\varphi)=-2 \cos (\pi \varphi)+32, f_{4}(\varphi)=126$. First we find $\varphi$ from the equation $\left|\begin{array}{ll}\gamma_{3} & \gamma_{5} \\ \gamma_{5} & \gamma_{7}\end{array}\right|=0$, where $\gamma_{1}=C_{1}, \gamma_{3}=\left(C_{1}-\gamma_{1}^{3}\right) / 3, \gamma_{5}=$ $\left(C_{5}-C_{3} \gamma_{1}^{2}-2 C_{1} \gamma_{1} \gamma_{3}\right) / 5, \gamma_{7}=\left(C_{7}-C_{5} \gamma_{1}^{2}-2 C_{3} \gamma_{1} \gamma_{3}-2 C_{1} \gamma_{1} \gamma_{5}-C_{1} \gamma_{3}^{2}\right) / 7, C_{1}=$ $4-\varphi^{2}, C_{3}=6-3 \sin \frac{\pi \varphi}{2}, C_{5}=-32+2 \cos \pi \varphi, C_{7}=-126$.

Let $\bar{\varphi}$ be one of the roots of this equation. Then the equation for the deter-

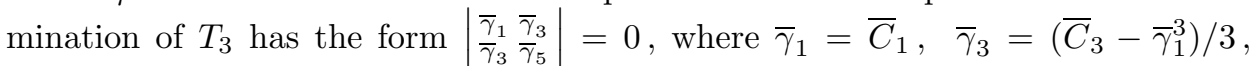
$\bar{\gamma}_{5}=\left(\bar{C}_{5}-\bar{C}_{3} \bar{\gamma}_{1}^{2}-2 \bar{C}_{1} \bar{\gamma}_{1} \bar{\gamma}_{3}\right) / 5$ and $\bar{C}_{1}=C_{1}-T_{3}=4-\bar{\varphi}^{2}-T_{3}, \bar{C}_{3}=C_{3}-T_{3}^{3}=$ $-6-3 \sin \frac{\bar{\pi} \varphi}{2}-T_{3}^{3}, \bar{C}_{5}=C_{5}-T_{3}^{5}=-32+2 \cos \bar{\pi} \varphi-T_{3}^{5}$.

Now let $\bar{T}_{3}$ be one of the solutions of this equation. The equation for finding $T_{1}$ and $T_{2}$ has the form $\widetilde{\gamma}_{3}(T)=0$, where $\widetilde{\gamma}_{1}=4-\bar{\varphi}^{2}-\widetilde{T}_{3}+T, \quad \widetilde{\gamma}_{3}=$ $\frac{1}{3}\left(-6-3 \sin \frac{\pi \bar{\varphi}}{2}-\widetilde{T}_{3}^{3}+T^{3}-\widetilde{\gamma}_{1}^{3}\right)$.

Finally, let $\bar{T}_{1}, \bar{T}_{2}$ be the solutions of the equation $\widetilde{\gamma}_{3}(T)=0$. Then from the equality $\bar{T}_{1}+T_{4}=\bar{\varphi}$ we get $T_{4}$.

For example, some solutions $\left(T_{1}, T_{2}, T_{3}, T_{4}\right)$ of system (4.16) are as follows: $(2 ;-1 ; 1 ; 0) ; \quad(-1 ;-1 ;-2 ; 3)$; $(-0.339987 \mp 1.06139 i ;-1.57683 \pm 0.952494 i ; 1.61404 \pm 0.95331 i ;-0.563 \pm 1.64966 i)$; $(0.676173 \pm 1.11377 i ; 0.676173 \mp 1.11337 i ;-1.9769 ; 2.031527 \mp 1.11377 i)$.

Remark 4.2. If we put $\varphi\left(T_{1}, \ldots, T_{n}\right)=T_{n}$, then we have $f_{2 k-1}\left(\varphi\left(T_{1}, \ldots, T_{n}\right)\right)=$ $f_{2 k-1}\left(T_{n}\right), k=1, \ldots, n$, hence solving the system (1.2) is reduced to finding the roots of three functions of one variable. If $f_{2 k-1}\left(\varphi\left(T_{1}, \ldots, T_{n}\right)\right)=T_{n}^{2 k-1}$, then solving system (1.2) is reduced to the search of the roots of two functions of one variable. 


\section{Solving Almost Power sum systems without gaps}

Now let us consider system (1.1) with an arbitrary number of positive and negative summands without gaps of the powers of $T_{i}$. We write it in the form

$$
-\sum_{i=1}^{m} T_{i}^{k}+\sum_{i=m+1}^{n-1} T_{i}^{k}=C_{k}, k=1, \ldots, n,
$$

where $C_{k}=-f_{k}\left(\varphi\left(T_{1}, \ldots, T_{n}\right)\right.$. Let us assume that $0 \leq 2 m \leq n-1$ (for $2 m>n-1$ it is sufficiently to multiply each equation of system (5.1) by -1 ). We complete system (5.1) by analogous equations for $k \geq n+1$, and we consider the infinite system of the equations (obtained in a similar way as (4.1) before)

$$
-\sum_{i=1}^{m} T_{i}^{k}+\sum_{i=m+1}^{n-1} T_{i}^{k}=C_{k}, \quad k=1,2, \ldots, \infty .
$$

Here, $C_{k}$ as $k>n$ are meaningless as in (4.1).

Let us consider the equality

$$
\ln \frac{\left(z-T_{m+1}\right)\left(z-T_{m+2}\right) \ldots\left(z-T_{n-1}\right)}{z^{n-2 m-1}\left(z-T_{1}\right)\left(z-T_{2}\right) \ldots\left(z-T_{m}\right)}=-\sum_{k=1}^{\infty} \frac{C_{k}}{k z^{k}} .
$$

As before, the infinite system of equations (5.2) is obtained from equality (5.3) with the aid of the formal expansion of the left-hand side of the equality (5.3) into Taylor series with respect to $1 / z$, comparing the coefficients with the same powers of $z$. We denote

$$
\begin{gathered}
R(z)=\frac{\left(z-T_{m+1}\right) \ldots\left(z-T_{n-1}\right)}{z^{n-2 m-1}\left(z-T_{1}\right) \ldots\left(z-T_{m}\right)} \\
=\frac{z^{n-m-1}-\widehat{\sigma}_{1} z^{n-m-2}+\cdots+(-1)^{n-m-2} \widehat{\sigma}_{n-m-2} z+(-1)^{n-m-1} \widehat{\sigma}_{n-m-1}}{z^{n-2 m-1}\left(z^{m}-\widetilde{\sigma}_{1} z^{m-1}+\cdots+(-1)^{m-1} \widetilde{\sigma}_{m-1} z+(-1)^{m} \widehat{\sigma}_{m}\right)},
\end{gathered}
$$

where $\widehat{\sigma}_{k}, k=1, \ldots, n-m-1, \widetilde{\sigma}_{k}, k=1, \ldots, m$ are defined in Section 4 .

The series expansion of the function $R(z)$ has the form

$$
R(z)=1-\sum_{k=1}^{\infty} \frac{\gamma_{k}}{z^{k}}
$$

where $\gamma_{k}, k=1,2, \ldots, \infty$, by virtue of (5.3) -(5.5), are defined by the recurrent relations

$$
\gamma_{1}=C_{1}, \quad \gamma_{k}=\frac{1}{k}\left(C_{k}-\sum_{i=1}^{k-1} \gamma_{i} C_{k-i}\right), \quad k=2,3, \ldots, \infty .
$$

From equalities (5.4) and (5.5) we have

$$
\begin{aligned}
z^{n-m-1}-\widehat{\sigma}_{1} z^{n-m-2}+\cdots+ & (-1)^{n-m-2} \widehat{\sigma}_{n-m-2} z+(-1)^{n-m-1} \widehat{\sigma}_{n-m-1} \\
=z^{n-2 m-1}\left(z^{m}-\widetilde{\sigma}_{1} z^{m-1}+\right. & \left.\cdots+(-1)^{m-1} \widetilde{\sigma}_{m-1} z+(-1)^{m} \widehat{\sigma}_{m}\right) \\
& \times\left(z^{n-2 m-1}-\gamma_{1} z^{n-2 m-2}-\gamma_{2} z^{n-2 m-3}-\cdots\right) .
\end{aligned}
$$

If we compare the coefficients with the same negative powers of $z$ in the equality (4.7), then (in analogy to (4.7)) we have the equality

$$
\Delta_{n-2 m, m+1}=0 \text {. }
$$

Now let $\varphi$ be one of the roots of the equation (5.7). Now we will find two functions of one variable, the roots of which are unknown values $T_{1}, \ldots, T_{m}$, and 
$T_{m+1}, T_{m+2}, \ldots, T_{n-1}$. We will find the equations for $T_{1}, \ldots, T_{m}$. The equation (5.1) is symmetric with respect to the variables $T_{1}, \ldots, T_{m}$. Therefore, we denote by $T$ any of those variables. Put $T=T_{m}$. We write system (5.1) in the form

$$
-\sum_{i=1}^{m-1} T_{i}^{k}+\sum_{i=m+1}^{n-1} T_{i}^{k}=C_{k}, \quad k=1, \ldots, n-1,
$$

where $C_{k}=-f_{k}(\bar{\varphi})+T^{k}$.

We define $\gamma_{k}, k=1, \ldots, n-1$, from the equation (5.6) where $C_{k}=-f_{k}(\bar{\varphi})-$ $T^{k}$. Since the system (5.8) has one less term than (5.2) we obtain the polynomial equation of variable $T$ in the form

$$
\Delta_{n-2 m+1, m}=0 .
$$

The roots of this equation are the unknowns $T_{1}, \ldots T_{m}$.

Now we will find the polynomial equation, the roots of which are unknown values $T_{m+1}, \ldots, T_{n-1}$. Let $T_{1}, \ldots, T_{m}$ be the roots of (5.9). We rewrite system (5.1) in the form

$$
\sum_{i=m+1}^{n-1} T_{i}^{k}=\bar{s}_{k}, \quad k=1, \ldots, n-m-1,
$$

where $\bar{s}_{k}=-f_{k}(\bar{\varphi})+\sum_{i=1}^{m} T_{i}^{k}$. Since this system has the form (1.3) then unknown $T_{m+1}, \ldots, T_{n-1}$ are roots of a polynomial equation (similar to the equation (2.6)), namely

$$
t^{n-m-1}-\sigma_{1} t^{n-m-2}+\cdots+(-1)^{n-m-1} \sigma_{n-m-1}=0,
$$

where $\sigma_{k}$ are determined from the equation (2.4), in which $S_{k}$ are given numbers $\bar{s}_{k}$.

We will get the equation (5.11) in another form, similar to (2.9). Put $T=T_{n-1}$. The system for finding $T_{m+1}, \ldots, T_{n-1}$, has the form

$$
\sum_{i=m+1}^{n-2} T_{i}^{k}=\bar{s}_{k}-T, \quad k=1, \ldots, n-m-1 .
$$

The polynomial for finding $T_{m+1}, \ldots, T_{n-1}$ is the equation (2.9), in which $S_{i}$ are given numbers $\bar{s}_{i}, i=1, \ldots, n-3$. Now we will find unknown $T_{n}$. Let $T_{1}, \ldots, T_{n-1}$ be roots of the equations (5.9) and (5.11). Finally we find $T_{n}$ from the equation

$$
\varphi\left(T_{1}, \ldots, T_{n-1}, T_{n}\right)=\bar{\varphi} .
$$

Remark 5.1. In the case when $f_{k}\left(\varphi\left(T_{1}, \ldots, T_{n-1}, T_{n}\right)\right)=T_{n}^{k}$ solving of the system (1.1) can be reduced to finding the roots of two polynomials.

Summarizing, we proved

Theorem 5.2. Suppose the equation $\Delta_{n-2 m, m+1}=0$ has a solution $\varphi$ and the equation (5.12) has a solution $T$, then solving the system (1.2) is reduced to the successive search of the roots of four functions of one variable:

- equations $\Delta_{n-2 m, m+1}=0$ for finding $\varphi$;

- polynomial equations $\Delta_{n-2 m+1, m}=0$ for $T_{1}, \ldots, T_{m}$;

- polynomial equations (5.11) $\bar{\varphi}$ for $T_{m+1}, \ldots, T_{n-1}$;

- equation (5.12) for $T_{n}$. 


\section{ACKNOWLEDGMENTS}

The authors would like to thank the anonymous reviewers for their valuable comments and suggestions.

\section{REFERENCES}

[1] U. J. J. Le Verrier, Sur les variations séculaires des éléments elliptiques des sept planètes principales, J. Math. Pures Appl., Sér. 1, T. 5 (1840), pp. 220-254.

[2] P. L. Chebyshev, Sur les quadratures, J. Math. Pures Appl., Sér. 2, Vol. 19 (1874), pp. $19-34$.

[3] S. N. Bernstein, Sur la formule de quadrature approche de Tchebycheff, C. R. Math. Acad. Sci. Paris, 203 (1936), pp. 1305-1306.

[4] F. G. Mehler, Bemerkungen zur Theorie der mechanischen Quadraturen, J. Reine Angew. Math., Vol. 1864 (1864), Issue 63 (Jan 1, 1864) (1864), pp. 152-157.

[5] J. L. Ullman, A class of weight functions that admit Tchebycheff quadrature, Michigan Math. J. 13 (1966), 417-423. MR0205463 (34 \#5290)

[6] V. I. Korobov and G. M. Sklyar, Time-optimality and the power moment problem (Russian), Mat. Sb. (N.S.) 134(176) (1987), no. 2, 186-206, 287; English transl., Math. USSR-Sb. 62 (1989), no. 1, 185-206. MR922415 (89b:93056)

[7] V. I. Korobov and G. M. Sklyar, The Markov moment problem on a minimally possible segment (Russian), Dokl. Akad. Nauk SSSR 308 (1989), no. 3, 525-528; English transl., Soviet Math. Dokl. 40 (1990), no. 2, 334-337. MR.1021106 (91g:44008)

[8] V. I. Korobov and G. M. Sklyar, The Markov moment min-problem and time optimality (Russian), Sibirsk. Mat. Zh. 32 (1991), no. 1, 60-71, 220, DOI 10.1007/BF00970159; English transl., Siberian Math. J. 32 (1991), no. 1, 46-55. MR.1112081 (92c:49026)

[9] V. I. Korobov, G. M. Sklyar, and V. V. Florinskiı̌, A polynomial of minimal degree for determining all switching moments in a time-optimal problem (Russian, with English, Russian and Ukrainian summaries), Mat. Fiz. Anal. Geom. 7 (2000), no. 3, 308-320. MR.1797005 (2001k:49008)

[10] V. I. Korobov and G. M. Sklyar, Markov power min-moment problem with periodic gaps, J. Math. Sci. 80 (1996), no. 1, 1559-1581, DOI 10.1007/BF02363927. Dynamical systems, 2. MR:1402038(97k:44012)

[11] L. Gosse and O. Runborg, Finite moment problems and applications to multiphase computations in geometric optics, Commun. Math. Sci. 3 (2005), no. 3, 373-392. MR2165014 (2006e:65260)

[12] L. Gosse and O. Runborg, Resolution of the finite Markov moment problem (English, with English and French summaries), C. R. Math. Acad. Sci. Paris 341 (2005), no. 12, 775-780, DOI 10.1016/j.crma.2005.10.009. MR2188876

[13] L. Gosse and O. Runborg, Existence, uniqueness, and a constructive solution algorithm for a class of finite Markov moment problems, SIAM J. Appl. Math. 68 (2008), no. 6, 1618-1640, DOI 10.1137/070692510. MR2424956(2009k:30041)

[14] Y. Wu and C. N. Hadjicostis, On solving composite power polynomial equations, Math. Comp. 74 (2005), no. 250, 853-868 (electronic), DOI 10.1090/S0025-5718-04-01710-7. MR2114652 (2005k:65099)

[15] V. I. Korobov and A. N. Bugaevskaya, The solution of one time-optimal problem on the basis of the Markov moment min-problem with even gaps, Mat. Fiz. Anal. Geom. 10 (2003), no. 4, 505-523. MR 2020824 (2004k:49076)

V.N. Karazin Kharkov National University, 4 Svoboda sq., 61022 Kharkov, Ukraine Current address: Institute of Mathematics, Szczecin University, Wielkopolska str. 15, 70-451 Szczecin, Poland

E-mail address: korobow@univ.szczecin.pl

Belgorod State University, 85 Pobeda str., 308015 Belgorod, Russia

E-mail address: bugaevskaya@bsu.edu.ru 